\title{
The different causes of brachial plexus injuries
}

\author{
Syed Asif Hasan ${ }^{1 *}$, Abdulrahman Saad Aljammaz ${ }^{2}$, Mosleh Motesh AlGhamdi ${ }^{3}$, \\ Mohamed Jasim ALQattan ${ }^{4}$, Abdulrahman Hussain Alsalman ${ }^{5}$, Wafaa Sulaiman Alhifzi ${ }^{6}$, \\ Hassan Ahmed Aljudia ${ }^{7}$, Mazen Jarallah Alqahtani ${ }^{8}$, Abdullrahman Mohammed Ali ${ }^{6}$, \\ Ahad Mohammed Almutairi ${ }^{9}$, Abrar Hasan Bukhamseen ${ }^{10}$
}

\author{
${ }^{1}$ Department of Orthopaedics, Jeddah National Hospital, Jeddah, Saudi Arabia \\ ${ }^{2}$ College of Medicine, Shaqra University, Shaqra, Saudi Arabia \\ ${ }^{3}$ Department of Orthopaedics, Dhurma General Hospital, Riyadh, Saudi Arabia \\ ${ }^{4}$ Department of Emergency Medicine, King Hamad University Hospital, Muharraq, Bahrain \\ ${ }^{5}$ Department of Orthopaedics, Prince Mohammed Bin Abdulaziz Hospital, Riyadh, Saudi Arabia \\ ${ }^{6}$ College of Medicine, King Khalid University, Abha, Saudi Arabia \\ ${ }^{7}$ College of Medicine, Al-Jouf University, Al-Jouf, Saudi Arabia \\ ${ }^{8}$ Department of Orthopaedics, King Khalid Hospital, Najran, Saudi Arabia \\ ${ }^{9}$ College of Medicine, Unaizah College of Medicine, Unaizah, Saudi Arabia \\ ${ }^{10}$ College of Medicine, Imam Abdulrahman Bin Faisal University, Dammam, Saudi Arabia
}

Received: 08 July 2021

Accepted: 23 July 2021

\author{
*Correspondence: \\ Dr. Syed Asif Hasan, \\ E-mail: drasifh@gmail.com
}

Copyright: (C) the author(s), publisher and licensee Medip Academy. This is an open-access article distributed under the terms of the Creative Commons Attribution Non-Commercial License, which permits unrestricted non-commercial use, distribution, and reproduction in any medium, provided the original work is properly cited.

\begin{abstract}
Many etiologies have been reported to account for injuries to the brachial plexus, peripheral nerves and trauma. Additionally, many diseases have also been reported to cause the injury with many different pathophysiologies. For instance, some diseases have been classified as the primary diseases of the peripheral nerves including hereditary neuropathy. In the same context, brachial plexus damage or injury might also result secondary to a systemic disease, leading to a significant peripheral nerve injury as in cases with most metabolic neuropathies, which may result secondary to renal insufficiency, diabetes, amyloidosis and many other diseases. Furthermore, toxic and iatrogenic causes were also reported as potential causes for brachial plexus injuries. However, traumatic events are the most commonly reported, owing to motorcycle accidents, being the most common etiology. Although evidence is now abundant regarding the etiology, further studies are needed to furtherly validate the evidence and for more specification of the etiology and the underlying mechanisms.
\end{abstract}

Keywords: Microsurgery, Etiology, Injury, Brachial plexus, Neurosurgery

\section{INTRODUCTION}

Estimates show that although the prevalence rate of brachial plexus injuries is very low. It can be associated with severe morbidities and unfavorable prognostic outcomes. However, as a result of the recent medical advances, successful reconstruction of these injuries has been associated with favorable outcomes. ${ }^{1,2}$ On the other hand, severe or complicated cases might be hard to manage. ${ }^{3,5}$ In addition, young men of working age are the most commonly affected and the injury can have a significant impact on their socioeconomic status, leading to serious disabilities. ${ }^{6,7}$ Besides, the injury might cause motor, sensory or both motor and sensory impairment of the upper limb. The management of these injuries requires the integration of serious efforts and expert clinicians and surgeons for adequate evaluation and 
processing of the surgical procedures. Many etiologies have been proposed for brachial plexus injuries among the different studies in the literature. ${ }^{6,7}$ Identification of these causes might help to increase the awareness of the public, rendering them to be more careful with adequate interventions. This can also help physicians acquire more knowledge about the anatomical preference of these injuries, aiding their knowledge to enhance the underlying outcomes. Therefore, in this literature review, we aimed to discuss the different causes of brachial plexus injuries according to the different studies in the literature and previous meta-analysis.

\section{METHODS}

We performed an extensive literature search of the Medline, Cochrane and EMBASE databases on 18 July 2021 using the medical subject headings (MeSH) or a combination of all possible related terms. This was followed by the manual search for papers in Google scholar and the reference lists of the initially included papers. Papers discussing causes of branchial plexus injuries were screened for relevant information. We did not pose any limits on date, language, age of participants or publication type.

\section{DISCUSSION}

\section{Systemic causes}

Many etiologies have been reported to account for injuries to the brachial plexus, peripheral nerves and trauma. Additionally, many diseases have also been reported to cause the injury with many different pathophysiologies. For instance, some diseases have been classified as the primary diseases of the peripheral nerves including hereditary neuropathy. ${ }^{8}$ In the same context, brachial plexus damage or injury might also result secondary to a systemic disease, leading to a significant peripheral nerve injury as in cases with most metabolic neuropathies, which may result secondary to renal insufficiency, diabetes, amyloidosis and many other diseases. ${ }^{9}$ Toxic neuropathy is also another cause of injury to the brachial plexus which might result secondary to the chronic excessive administration of alcohol. ${ }^{10}$ Nutritional deficiency, as hypovitaminosis might also lead to the development of neuropathies and nerve damage. ${ }^{11}$ Injury to the brachial plexus might also occur secondary to the presence of serious inflammation owing to an infectious disease, like Lyme disease and HIV. ${ }^{12}$ Moreover, iatrogenic causes are also commonly reported to induce a significant injury to the peripheral nerves including the brachial plexus and some of the reported causes include post-actinic neuropathy and following chemotherapy. ${ }^{12}$ The pathophysiology of most of these etiologies is usually attributable to autoimmune reactions including both acute and chronic events as Guillain-Barrè syndrome and chronic inflammatory demyelinating polyneuropathies, respectively. ${ }^{13}$ Some injuries might be characterized by sensory or motor affection or both. Multifocal motor neuropathy is a significant example of motor-only affection. The nature of these diseases is usually systemic and the brachial plexus has been commonly observed to be affected in such situations. Although we did not aim to discuss the clinical manifestations of such injuries, we would like to indicate that they should be adequately assessed during the physical examination to establish a proper diagnosis.

\section{Surgical causes}

Among the reported etiologies, iatrogenic nerve injury was also a common condition that might unintentionally result from therapeutic and interventional surgical procedures. ${ }^{14}$ Injuries to the peripheral nerves and the brachial plexus in such situations are commonly reported, especially within clinics that are involved in sports practice. This was mainly attributable to frequently conducting surgical orthopedic procedures that were usually in direct association with the peripheral nerves and brachial plexus, as shoulder dislocation reduction and shoulder general surgeries. Neoplastic nerve injuries were also common causes of damage to the brachial plexus and two types had been reported, including neurinoma or primary neoplastic injuries and lung tumors-induced metastasis or direct affection to the brachial plexus or secondary neoplastic injuries. ${ }^{15}$ Therefore, adequate and proper examination of the patient and retrieving a full history was essential to establish a proper diagnosis and enhance the management outcomes. It should be noted, however, that the systemic and local diseases causing injuries to the brachial plexus were rare events. Brachial plexus injuries were mainly attributable to traumatic events and isolated brachial plexus injuries, according to various epidemiological investigations. ${ }^{16}$ In this context, previous studies have reported that men of the workingage were usually at more risk of having a brachial plexus injury, with an estimated female to male ratio of 1:13.3, respectively, indicating that these injuries were usually attributable to traumatic events. ${ }^{17}$

\section{Traumatic causes}

Besides, it was also previously estimated that $93 \%$ of the etiologies causing traumatic injuries to the brachial plexus were closed lesions, which are usually associated with tractions of the peripheral nerves. ${ }^{18}$ Other etiologies for traumatic events included lacerations, gunshot lesions and iatrogenic etiologies. Estimates also showed that the complete involvement of the brachial plexus was commonly secondary to traumatic events, with a prevalence rate of $53 \%$. However, it was also observed that injuries to the upper structures were more common than the lower ones. ${ }^{17}$ Furthermore, trunks and roots of the brachial plexus (supraclavicular structures) have also been reported to be more common in these situations. The most common traumatic etiology causing brachial plexus injuries was the injuries following motorcycle accidents, owing to a total of $67 \%$ from the traumatic injuries. ${ }^{17}$ In such traumatic events, the injury was usually severe and was usually associated with the velocity of the motorcycle, on which the clinical presentation of the affected patients depends. ${ }^{16}$ Sports or occupational 
activities have been estimated to cause around $10 \%$ of the total traumatic events causing brachial plexus injuries. ${ }^{17}$ Previous estimates also showed that the incidence of brachial plexus injury following winter sporting events was $4 \% .{ }^{19}$ Although contact sports are very common to cause injuries to the brachial plexus, the exact epidemiology of such events was hard to be estimated. Burners and stingers syndrome or lesions that were associated with neuropraxia were also common among sporting activities and are usually associated with a significant effect on the supraclavicular region, namely kick in martial arts. ${ }^{16}$ However, because these injuries are usually associated with a complete functional recovery, estimating the exact incidence and prevalence rates was hard in such situations, leading to the reporting of underestimated statistics.

This concept should also be considered with some sporting activities as a result of the huge development of the current interventional approaches in these sports. A compelling example of this was the traumatic injuries related to the practice of American football. Although using helmets was associated with a significant reduction in concussion events, estimates showed that supraclavicular and shoulder injuries are commonly reported in these situations with more frequent injuries to the brachial plexus. ${ }^{20}$ In the same context, using helmets for interventions against motorcycle injuries has been more significantly associated with more proper management of the related traumatic events, which has been associated with higher survival rates and enhanced prognosis of brachial plexus injuries. ${ }^{21}$ Bias in reporting should be considered when interpreting these statistics that are mainly reported to the sporting activities. The majority of reports that estimated the epidemiology of the traumatic brachial plexus injuries were mainly attributable to cases that require surgical management only. ${ }^{22}$ On the other hand, many cases with brachial plexus injury have been associated with milder forms and reported partial axonotmesis and neurapraxia only, which were usually associated with complete functional recovery following the administration of conservative management modalities. Therefore, many studies have not estimated these events in their reports, leading to a potential underestimation of the true prevalence and incidence of brachial plexus injuries. Besides, many reports have shown that the incidence and prevalence of mild lesions related to the brachial plexus were more common than the rates reported for the severe lesions, indicating the potential underestimation of the true epidemiological data regarding traumatic brachial plexus injuries. For instance, burners and stingers syndrome was previously estimated to occur in up to $30-40 \%$ of rugby players. ${ }^{23} \mathrm{~A}$ previous football study in Canada also showed that around $26 \%$ of the included participants suffered from stingers and only $59 \%$ of them were properly referred to be evaluated and managed within a clinical setting. ${ }^{24}$ Having a previous history of a stinger and the duration of practicing sporting activities were usually associated with a sustained stinger. In this context, a previous estimate related to American football indicated that more than half of the participants suffer from stingers related to brachial plexus injuries.
Besides, the authors also reported that the incidence of the injury has been estimated to be associated with the role of the player in his team. For instance, defensive linemen and running backs have been reported with higher incidence rates of brachial plexus injuries. ${ }^{25}$ Accordingly, when interpreting the prevalence and incidence of the traumatic etiologies these points should be considered.

It was worth mentioning that the current evidence showed that the etiology of brachial plexus injuries has significantly changed over the years, as open injuries were the most common during the past, when wars were common, while recently, most injuries were closed ones. ${ }^{26}$ Among studies that reported that the prevalence of closed injuries, the rates were hugely variable from $72.9 \%$ to $99.3 \%$ and Kaiser et al estimated a pooled prevalence of $93 \%$ among the different studies in the literature. 1,17,27 Traction and compression were the two mechanisms that can lead to the development of a closed injury. ${ }^{28}$ Traffic accidents were the commonest causes of these injuries. However, falls were also reported as potential causes. Besides, it has also been observed that if a severe force was applied. It can lead to the development of a complete lesion which can also happen in cases when several forces are applied to a single region. The previous meta-analysis by Kaiser et al also estimated a pooled prevalence of $67 \%$ for motorcycle accidents, being the commonest etiology for brachial plexus injuries. ${ }^{17}$ Besides, a previous investigation also reported that the dominant limb was the most commonly affected during these accidents regardless of the traffic side. ${ }^{27}$ Different speeds of the motorcycles were also variably reported during the time of the accident, ranging between 88 and $47 \mathrm{~km} / \mathrm{h}$ in the United States and India, respectively. ${ }^{27,29}$ Car accidents were also reported as potential causes of brachial plexus injuries, however, they were less commonly reported, with an estimated prevalence rate of $14 \%$ among studies in the literature. ${ }^{17}$ Pedestrian or bicycle accidents were also reported, however, these were very rare causes among studies. There was still an urgent need to furtherly specify the etiologies causing infra and supraclavicular lesions and identify the appropriate mechanisms for each to enhance the outcomes of these lesions.

\section{CONCLUSION}

Metabolic etiologies can be either primary as a disease affecting the peripheral nerves or secondary as a part of a complication of systemic disease. Toxic and iatrogenic causes were also reported as potential causes for brachial plexus injuries, however, traumatic events are the most commonly reported, owing to motorcycle accidents, being the most common etiology. Although evidence is now abundant regarding the etiology, further studies are needed to furtherly validate the evidence and for more specification of the etiology and the underlying mechanisms. 
Funding: No funding sources

Conflict of interest: None declared

Ethical approval: Not required

\section{REFERENCES}

1. Kim DH, Cho YJ, Tiel RL, Kline DG. Outcomes of surgery in 1019 brachial plexus lesions treated at Louisiana state university health sciences center. J Neurosurg. 2003;98(5):1005-16.

2. Songcharoen P. Brachial plexus injury in Thailand: a report of 520 cases. Microsurgery. 1995;16(1):359.

3. Lanaras TI, Schaller HE, Sinis N. Brachial plexus lesions: 10 years of experience in a center for microsurgery in Germany. Microsurgery. 2009;29(2):87-94.

4. Johnson EO, Vekris MD, Zoubos AB, Soucacos PN. Neuroanatomy of the brachial plexus: the missing link in the continuity between the central and peripheral nervous systems. Microsurgery. 2006;26(4):218-29.

5. Vekris MD, Beris AE, Johnson EO, Korobilias AV, Pafilas D, Vekris AD, et al. Musculocutaneous neurotization to restore elbow flexion in brachial plexus paralysis. Microsurgery. 2006;26(4):325-9.

6. Ahmed-Labib M, Golan JD, Jacques L. Functional outcome of brachial plexus reconstruction after trauma. Neurosurgery. 2007;61(5):1016-22.

7. Krishnan KG, Martin KD, Schackert G. Traumatic lesions of the brachial plexus: an analysis of outcomes in primary brachial plexus reconstruction and secondary functional arm reanimation. Neurosurgery. 2008;62(4):873-85.

8. Eggermann K, Gess B, Häusler M, Weis J, Hahn A, Kurth I. Hereditary Neuropathies. Dtsch Arztebl Int. 2018;115(6):91-7.

9. Callaghan B, Feldman E. The metabolic syndrome and neuropathy: therapeutic challenges and opportunities. Ann Neurol. 2013;74(3):397-403.

10. Manji H. Toxic neuropathy. Curr Opin Neurol. 2011;24(5):484-90.

11. Hammond N, Wang Y, Dimachkie MM, Barohn RJ. Nutritional neuropathies. Neurol Clin. 2013;31(2):477-89.

12. Freitas MRD. Infectious neuropathy. Curr Opin Neurol. 2007;20(5):548-52.

13. Ho TW, McKhann GM, Griffin JW. Human autoimmune neuropathies. Ann Rev Neurosci. 1998;21:187-226.

14. Toyooka K, Fujimura H. Iatrogenic neuropathies. Curr Opin Neurol. 2009;22(5):475-9.

15. Koike H, Tanaka F, Sobue G. Paraneoplastic neuropathy: wide-ranging clinicopathological manifestations. Curr Opin Neurol. 2011;24(5):50410.
16. Clancy WG, Brand RL, Bergfield JA. Upper trunk brachial plexus injuries in contact sports. Am J Sports Med. 1977;5(5):209-16.

17. Kaiser R, Waldauf P, Ullas G, Krajcová A. Epidemiology, etiology, and types of severe adult brachial plexus injuries requiring surgical repair: systematic review and meta-analysis. Neurosurg Rev. 2020;43(2):443-52.

18. Sakellariou VI, Badilas NK, Mazis GA, Stavropoulos NA, Kotoulas HK, Kyriakopoulos S, et al. Brachial plexus injuries in adults: evaluation and diagnostic approach. ISRN Orthop. 2014;2014:726103.

19. Kaiser R, Mencl L, Haninec P. Injuries associated with serious brachial plexus involvement in polytrauma among patients requiring surgical repair. Injury. 2014;45(1):223-6.

20. Gorden JA, Straub SJ, Swanik CB, Swanik KA. Effects of football collars on cervical hyperextension and lateral flexion. J Athl Train. 2003;38(3):209-15.

21. Ferraresi S, Garozzo D, Griffini C, Resmini B, Manara O, Foresti C, et al. Brachial plexus injuries. Guidelines for management: our experience. Ital $\mathbf{J}$ Neurol Sci. 1994;15(6):273-84.

22. Arzillo S, Gishen K, Askari M. Brachial plexus injury: treatment options and outcomes. J Craniofac Surg. 2014;25(4):1200-6.

23. Kawasaki T, Maki N, Shimizu K, Ota C, Urayama $\mathrm{S}$, Moriya S, et al. Do stingers affect scapular kinematics in rugby players? J Shoulder Elbow Surg. 2014;23(12):293-9.

24. Charbonneau RM, McVeigh SA, Thompson K. Brachial neuropraxia in Canadian Atlantic University sport football players: what is the incidence of "stingers"? Clinic J Sport Med. 2012;22(6):472-7.

25. Feinberg JH. Burners and stingers. Phys Med Rehab Clinics North Am. 2000;11(4):771-84.

26. Kawai H, Kawabata H. Brachial plexus palsy. Osaka: World Scientific; 2000.

27. Jain DK, Bhardwaj P, Venkataramani H, Sabapathy SR. An epidemiological study of traumatic brachial plexus injury patients treated at an Indian centre. Indian J Plastic Surg. 2012;45(3):498-503.

28. Midha R. Epidemiology of brachial plexus injuries in a multitrauma population. Neurosurgery. 1997;40(6):1182-8.

29. Terzis JK, Vekris MD, Soucacos PN. Outcomes of brachial plexus reconstruction in 204 patients with devastating paralysis. Plast Reconstr Surg. 1999;104(5):1221-40.

Cite this article as: Hasan SA, Aljammaz AS, AlGhamdi MM, ALQattan MJ, Alsalman AH, Alhifzi WS, et al. The different causes of brachial plexus injuries. Int J Community Med Public Health 2021;8:4119-22. 